\title{
13/9 Lifting Wavelet Transform based Reversible Data Hiding
}

\author{
Subash N. \\ Research Scholar, RGMCET \\ JNTUA, Ananthapuramu, \\ Andhra Pradesh, India
}

\author{
Jayachandra Prasad T. \\ Dept of ECE, RGMCET \\ JNTUA, Ananthapuramu \\ Andhra Pradesh, India
}

\author{
Sumalatha V. \\ Dept of ECE \\ JNTUA College of Engineering \\ Ananthapuramu, Andhra \\ Pradesh, India
}

\begin{abstract}
In this paper a novel 13/9 lifting scheme for hiding data has been implemented. Lifting schemes are the part of wavelet transformation which decomposes image into wavelet coefficients using basis function for analyzing multiresolution images. Lifting scheme is preferred for data hiding because the operations are performed in spatial domain by simple LSB replacement. The 13/9 lifting scheme contains 13 low pass and 9 high pass coefficients. As more information in image lies at low frequency components, 13/9 implemented lifting scheme contains 13 low-frequency components, whereas 5/3 lifting scheme contains 5 low frequencies and 9/7 lifting scheme contains 9 low frequencies. Simulation results show that the payload and average PSNR are increased than the existing methods with the PSNR $58.17 \mathrm{~dB}$ at bit rate of 0.05 for Lena image.
\end{abstract}

\section{General Terms}

Image Processing, Security

\section{Keywords}

Reversible Data Hiding, Encryption, Lifting Wavelets, Steganography.

\section{INTRODUCTION}

With the rapid change in communication media maintaining security and safety became a challenging task. Recently attention was drawn to attain reversibility in images known as Reversible Data Hiding (RDH) which is branch of steganography. It hides data, images, audio or video formats into another text, image, audio or video formats. It aims at recovering the input image from the data hidden image when secret message is extracted. Due to this RDH became interesting area for the researchers in the field of image processing. They provided different algorithms for the improvement of the image quality and attaining reversibility. These methodologies are categorized into Spatial and Transform domain approaches. In spatial domain, one of the simplest technique is least significant bit (LSB) replacement. Based on this process RDH schemes are further classified into four types: 1) Difference Expansion 2) Histogram method 3) prediction methods 4) Interpolation Algorithms.

Tian [1] introduced difference expansion method with high capacity and less distortion. The disadvantage is binary map size was not known and low compressibility of the location map. To over this problem Alattar [2] used vectors as difference expansion and improved capacity. $\mathrm{Hu}$ et al. [3] presents a variant DE technique which improved the compressibility of the location map with increase in payload for different image types. In 2009 Yuan et al. [4] implemented an algorithm to hide data into embedding area and secondary information areas in which data was hidden in embedding areas and overhead bits are inserted into secondary information area. Ni et al. [5] implemented data hiding based on histogram by calculating minimum and maximum points and made some modification to the pixel gray values to embed data and proved that this method can embed data up to $80 \mathrm{~kb}$ while maintaining high visual quality than existing methods. Tseng and Hsieh [6] proposed two methods, in the first method histogram was taken for sub-block while maximum points remain unmodified to pull out the embedded data and in the second method to decrease the overhead information size shifting operation is performed which improves the image quality $>48 \mathrm{~dB}$. Thodi and Rodriguez [7] used both DE and histogram methods, and developed a new technique by reducing the distortion occurred in difference expansion to attain reversibility. W.L.Tai et al. [8] proposed novel method on histogram modification using binary tree method and improved the payload with low distortion.

Researchers later combined histogram based methods with prediction methods. 2008 Piyu Tsai et al. [9] proposed an RDH method on predictive coding and improved the hiding capacity, which is three times larger than the normal histogram method with improved image quality of $1.5 \mathrm{~dB}$. Vasiliy et al. [10] used technique of sorting and prediction errors for data embedding to attain low distortion and the histogram shift scheme was implemented for decreasing the location map size and increase the capacity further. Chin-Feng et al. [11] implemented location map free region for input image recovery with minimal visual distortion. Xiaolong Li et al. [12] proposed a novel method for hiding data using pixel-value-ordering to improve the performance in PSNR. Bo et al. [13] applied partial differential equation predictor function for sub-blocks which gives less distortion.

Lixin Luo et al. [14] worked in the sub-domain of interpolation in $\mathrm{RDH}$ by embedding data into variation between the interpolation error and corresponding pixel values and achieved payload of 84,050 bits with PSNR of 48dB. Wang et al. [15] proposed RDH technique in spatial domain for enhancing the image quality by classifying elements as wall and non-wall pixels. Yalman and Akar [16] proposed a novel method for hiding and increased payload up to $6,02,406$ bits at rate of $0.766 \mathrm{bpp}$ for color image of Lena with PSNR $42.43 \mathrm{~dB}$ and further data hiding capacity can be increased by applying NMI more than once.

Additionally, focusing on transformation techniques for data hiding has improved the payload with high PSNR and many researchers provided and extended research in that area. Lin and Shiu [17] implemented a novel hiding method which is notation based for DCT images with high image quality. Fei Peng et al. [18] implemented an adaptive embedding using integer transform for hiding data in smooth blocks and 
improved PSNR. Yang et al. [19] implemented integer wavelet transform for hiding data in LH and HL sub-bands and attained a high average payload of 78,643 bits with average PSNR of $49.49 \mathrm{~dB}$. Lin [20] implemented DCT and used high frequency components to embed secret data and obtained payload of $1,52,389$ bits with PSNR $36.44 \mathrm{~dB}$ for 8x8 block size. Engin Avci et al. [21] presented a wavelet domain based method and enhanced the visual quality to $52.16 \mathrm{~dB}$ with payload capacity of 1,17,160 bits. Fan Li et al. [22] implemented a RDH scheme in frequency domain using Haar Transform and improved the performance in PSNR and payload. Kede Ma et al. [23] proposed method for hiding data in encrypted images and attained reversibility.

In this paper, we implemented a 13/9 lifting scheme by embedding data into the Inverse transformed images and observed that this method performs well in payload and average PSNR when compared to existing techniques. From the simulation results, our 13/9 lifting method attains high PSNR when compared to other methods, such as Ni et al [5], Thodi and Rodriguez [7], Tai et al [8] and Kede Ma et al [23].

The broad categories of the paper are as follows: Section 2 described about $5 / 3$ and $9 / 7$ lifting schemes. Section 3 presents algorithm for 13/9 lifting scheme and embedded data into it. In section 4 we present the Simulation results from which necessary Conclusions are drawn in Section 5.

\section{RELATED WORK}

Wim Swelden developed lifting concept in spatial domain. It breaks the poly phase matrices into a sequence of superior and inferior matrices. A new lifting scheme was constructed using bi-orthogonal wavelets with the poly phase matrix as:

$$
P(z)=\left[\begin{array}{ll}
h_{e} & g_{e} \\
h_{o} & g_{o}
\end{array}\right]
$$

The factorizations aim at representing the poly phase matrix as a set of superior and inferior matrices as [24]:

$$
P(z)=\prod_{i=1}^{m}\left[\begin{array}{cc}
1 & S_{i}(z) \\
0 & 1
\end{array}\right]\left[\begin{array}{cc}
1 & 0 \\
t_{i}(z) & 1
\end{array}\right]\left[\begin{array}{cc}
K & 1 \\
1 & 1 / K
\end{array}\right]
$$

Where K- Scaling factor.

$$
S_{i}(z), t_{i}(z) \text { - polynomials with primal and dual lifting }
$$
stages

The main steps involved in lifting scheme are: Splitting, Prediction and Updating.

The different lifting scheme based wavelets existing in literature are $5 / 3$ and $9 / 7$.

\subsection{5/3 Lifting Scheme:}

$5 / 3$ lifting scheme was introduced by LeGall. It contains 3 high pass and 5 low pass tap filters. This is the simplest process of decomposing the image into high and lowfrequency components. It contains two vanishing moments.
The lifting factors of $P(z)$ are given below:

$$
P(z)=\left(\left[\begin{array}{cc}
1 & 0 \\
\frac{1}{2}(1+z) & 1
\end{array}\right]\left[\begin{array}{cc}
1 & -\frac{1}{4}\left(1+z^{-1}\right) \\
0 & 1
\end{array}\right]\right)\left[\begin{array}{ll}
1 & 0 \\
0 & 2
\end{array}\right]
$$

The filter coefficients for 5/3 lifting scheme [25] are:

$$
\begin{aligned}
& H(n)=x(2 n+1)+\frac{1}{4}(x(2 n)+x(2 n+2)) \\
& L(n)=x(2 n)-\frac{1}{2}(H(n)+H(n-1))
\end{aligned}
$$

Where $\mathrm{H}$ and $\mathrm{L}$ are maximum and minimum frequency components. The performance of $5 / 3$ lifting scheme is better in PSNR than other existing wavelets where as poor compression ratio or the hiding capacity is low. To increase embedding rate $9 / 7$ lifting scheme was designed.

\subsection{9/7 Lifting Scheme:}

The algorithm designed for JPEG2000 standards uses a set of 9 low pass and 7 high pass filter coefficients with 4 vanishing moments in image compression domain. The filter is factorized as follows [24]:

$$
\begin{aligned}
P(z)= & {\left[\begin{array}{cc}
1 & \alpha\left(1+z^{-1}\right) \\
0 & 1
\end{array}\right]\left[\begin{array}{cc}
1 & 0 \\
\beta(1+z) & 1
\end{array}\right]\left[\begin{array}{cc}
1 & \gamma\left(1+z^{-1}\right) \\
0 & 1
\end{array}\right] } \\
& {\left[\begin{array}{cc}
1 & 0 \\
\delta(1+z) & 1
\end{array}\right]\left[\begin{array}{cc}
\varsigma & 0 \\
0 & \frac{1}{\varsigma}
\end{array}\right] }
\end{aligned}
$$

Where $\alpha, \beta, \gamma, \delta$ - are filter coefficients,

$$
\checkmark \text { - Scaling factor }
$$

It is found that the $9 / 7$ methodology is more efficient due to better scaling factor with increase in the payload size when compared to $5 / 3$ lifting scheme but visual quality has degraded. So achieving high image quality with high payload became a challenging task. In order to address this issue a new 13/9 lifting scheme is proposed which can overcome the design drawback of the earlier lifting schemes.

\section{PROPOSED METHOD}

From the previous methods of lifting schemes like $5 / 3$ or $9 / 7$, there is a conflict that either image quality or embedding capacity will be high but both cannot be achieved. Here we are implementing a novel 13/9 lifting scheme and embed data to attain reversibility with better visual quality and high payload. The main advantages in lifting scheme compared to other wavelets are: It requires very less computation, easy to perform, independent of Fourier transform, time-efficient. The four steps involved in implementation are: 1) Algorithm for 13/9 lifting scheme 2) Algorithm for encryption 3) Data Hiding Algorithm 4) Extraction Algorithm. The schematic representation is shown in Fig.1. 


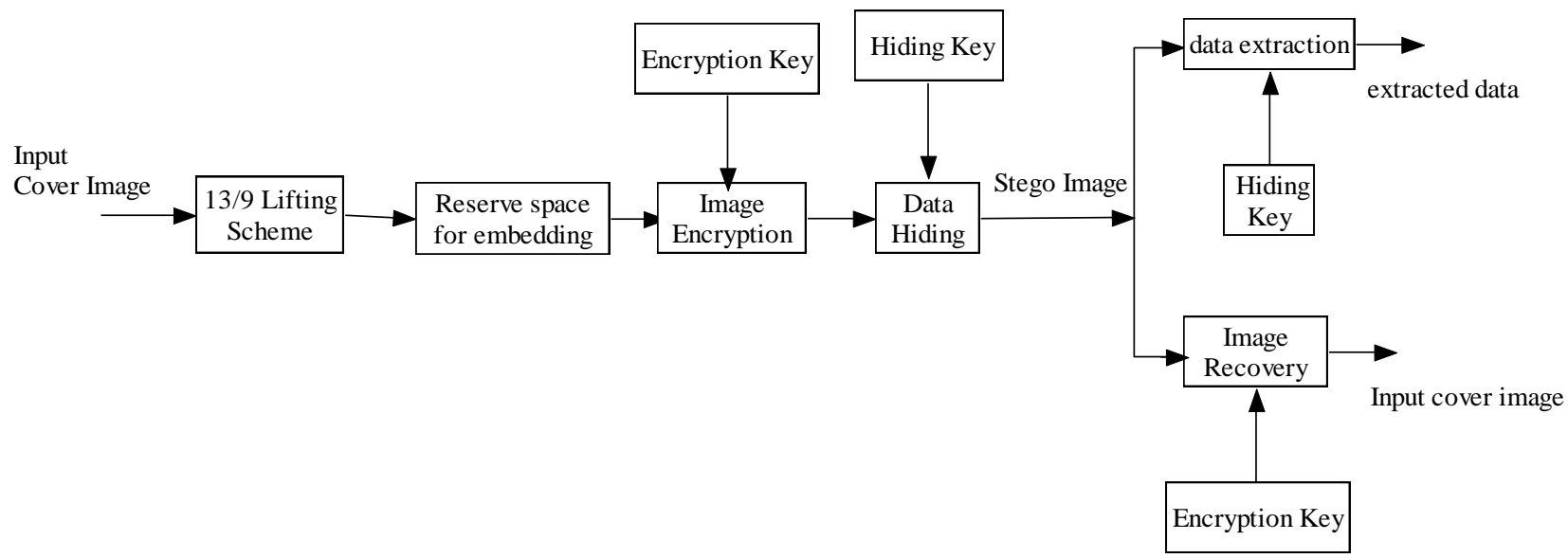

Fig 1: Frame work implementation

\subsection{Algorithm for 13/9 Lifting Scheme}

The algorithm for $13 / 9$ scheme is presented. This algorithm contains two phases: forward and reverse transform.

\subsubsection{Algorithm for forward transform}

The algorithm for forward transform contains both: Row transformation and Column transformation.

i) Row transformation:

Consider $\mathrm{r}$ as the row number, $\mathrm{c}$ as column number and $\mathrm{p}$ as $\mathrm{c}-1$.

a) For every row

The first column

$$
\begin{aligned}
& \mathrm{lo}() \leftarrow 2(\mathrm{r}, 2 \mathrm{c}-1)+6(\mathrm{r}, 2 \mathrm{c})+2(\mathrm{r}, 2 \mathrm{c}+1)-(\mathrm{r}, 2 \mathrm{c}+2) \\
& \mathrm{hi}() \leftarrow 2(\mathrm{r}, 2 \mathrm{p}+1)-(\mathrm{r}, 2 \mathrm{p}+2)
\end{aligned}
$$

While last column

$$
\begin{aligned}
& \text { lo }() \leftarrow-(r, 2 c-2)+2(r, 2 c-1)+6(r, 2 c) \\
& \text { hi }() \leftarrow-(r, 2 p)+2(r, 2 p+1)-(r, 2 p+2)
\end{aligned}
$$

and remaining columns

$$
\begin{aligned}
& \text { lo }() \leftarrow-(r, 2 c-2)+2(r, 2 c-)+6(r, 2 c)+2(r, 2 c+1)- \\
& (r, 2 c+2) \\
& \text { hi }() \leftarrow-(r, 2 p)+2(r, 2 p+1)-(r, 2 p+2)
\end{aligned}
$$

ii)

$$
\begin{aligned}
& \text { Column Transformation } \\
& \text { a. } \quad \text { For each column } \\
& \text { The first row } \\
& \mathrm{a}() \leftarrow 2(2 \mathrm{r}-1, \mathrm{c})+6(2 \mathrm{r}, \mathrm{c})+2(2 \mathrm{r}+1, \mathrm{c})-(2 \mathrm{r}+2, \mathrm{c}) \\
& \mathrm{h}() \leftarrow 2(2 \mathrm{p}+1, \mathrm{c})-(2 \mathrm{p}+2, \mathrm{c}) \\
& \mathrm{v}() \leftarrow 2(2 \mathrm{r}-1, \mathrm{c})+6(2 \mathrm{r}, \mathrm{c})+2(2 \mathrm{r}+1, \mathrm{c})-(2 \mathrm{r}+2, \mathrm{c}) \\
& \mathrm{d}() \leftarrow 2(2 \mathrm{p}+1, \mathrm{c})-(2 \mathrm{p}+2, \mathrm{c}) \\
& \mathrm{While} \text { last row } \\
& \mathrm{a}() \leftarrow-(2 \mathrm{r}-2, \mathrm{c})+2(2 \mathrm{r}-1, \mathrm{c})+6(2 \mathrm{r}, \mathrm{c}) \\
& \mathrm{h}() \leftarrow-(2 \mathrm{p}, \mathrm{c})+2(2 \mathrm{p}+1, \mathrm{c})-(2 \mathrm{p}+2, \mathrm{c}) \\
& \mathrm{v}() \leftarrow-(2 \mathrm{r}-2, \mathrm{c})+2(2 \mathrm{r}-1, \mathrm{c})+6(2 \mathrm{r}, \mathrm{c}) \\
& \mathrm{d}() \leftarrow-(2 \mathrm{p}, \mathrm{c})+2(2 \mathrm{p}+1, \mathrm{c})-(2 \mathrm{p}+2, \mathrm{c})
\end{aligned}
$$

and remaining rows

$$
\begin{aligned}
& \mathrm{a}() \leftarrow-(2 \mathrm{r}-2, \mathrm{c})+2(2 \mathrm{r}-1, \mathrm{c})+6(2 \mathrm{r}, \mathrm{c})+2(2 \mathrm{r}+1, \mathrm{c})- \\
& \quad(2 \mathrm{r}+2, \mathrm{c}) \\
& \mathrm{h}() \leftarrow-(2 \mathrm{p}, \mathrm{c})+2(2 \mathrm{p}+1, \mathrm{c})-(2 \mathrm{p}+2, \mathrm{c}) \\
& \mathrm{v}() \leftarrow-(2 \mathrm{r}-2, \mathrm{c})+2(2 \mathrm{r}-1, \mathrm{c})+6(2 \mathrm{r}, \mathrm{c})+2(2 \mathrm{r}+1, \mathrm{c})-(2 \mathrm{r}+2, \mathrm{c}) \\
& \mathrm{d}() \leftarrow-(2 \mathrm{p}, \mathrm{c})+2(2 \mathrm{p}+1, \mathrm{c})-(2 \mathrm{p}+2, \mathrm{c})
\end{aligned}
$$

In the column transform ' $a$ ' and ' $v$ ' holds the filter samples of low pass where as ' $h$ ' $\&$ ' $d$ ' holds high pass filter samples.

\subsubsection{Algorithm for reverse transform}

Here for the column transform, the components a and $\mathrm{h}$ are combined to $\mathrm{X}$ and the components $\mathrm{v}$ and $\mathrm{d}$ combined to $\mathrm{Y}$. Finally $\mathrm{X}$ and $\mathrm{Y}$ are combined to $\mathrm{W}$.

i) Inverse Column transformation

For each column

The first row

$$
\begin{aligned}
& \mathrm{m}\left(1^{\text {st }} \text { Half }\right) \leftarrow 6 \mathrm{X}(2 \mathrm{r}-1, \mathrm{c})+2 \mathrm{X}(2 \mathrm{r}, \mathrm{c})-\mathrm{X}(2 \mathrm{r}+1, \mathrm{c}) \\
& \mathrm{m}\left(2^{\text {nd }} \text { Half }\right) \leftarrow-\mathrm{X}(2 \mathrm{r}-1, \mathrm{c})+2 \mathrm{X}(2 \mathrm{r}, \mathrm{c})-\mathrm{X}(2 \mathrm{r}+1, \mathrm{c}) \\
& \mathrm{n}\left(1^{\text {st }} \text { Half }\right) \leftarrow 6 \mathrm{Y}(2 \mathrm{r}-1, \mathrm{c})+2 \mathrm{Y}(2 \mathrm{r}, \mathrm{c})-\mathrm{Y}(2 \mathrm{r}+1, \mathrm{c}) \\
& \mathrm{n}\left(2^{\text {nd }} \text { Half }\right) \leftarrow-\mathrm{Y}(2 \mathrm{r}-1, \mathrm{c})+2 \mathrm{Y}(2 \mathrm{r}, \mathrm{c})-\mathrm{Y}(2 \mathrm{r}+1, \mathrm{c})
\end{aligned}
$$

While last row

$$
\begin{gathered}
\mathrm{m}\left(1^{\text {st }} \text { Half }\right) \leftarrow-\mathrm{X}(2 \mathrm{r}-3, \mathrm{c})+2 \mathrm{X}(2 \mathrm{r}-2, \mathrm{c})+6 \mathrm{X}(2 \mathrm{r}- \\
1, \mathrm{c})+2 \mathrm{X}(2 \mathrm{r}, \mathrm{c}) \\
\mathrm{m}\left(2^{\text {nd }} \text { Half }\right) \leftarrow-\mathrm{X}(2 \mathrm{r}-1, \mathrm{c})+2 \mathrm{X}(2 \mathrm{r}, \mathrm{c}) \\
\mathrm{n}\left(1^{\text {st }} \text { Half }\right) \leftarrow-\mathrm{Y}(2 \mathrm{r}-3, \mathrm{c})+2 \mathrm{Y}(2 \mathrm{r}-2, \mathrm{c})+6 \mathrm{Y}(2 \mathrm{r}- \\
1, \mathrm{c})+2 \mathrm{Y}(2 \mathrm{r}, \mathrm{c}) \\
\mathrm{n}\left(2^{\text {nd }} \text { Half }\right) \leftarrow-\mathrm{Y}(2 \mathrm{r}-1, \mathrm{c})+2 \mathrm{Y}(2 \mathrm{r}, \mathrm{c})
\end{gathered}
$$

and remaining rows

$$
\begin{aligned}
& \mathrm{m}\left(1^{\text {st }} \text { Half }\right) \leftarrow-\mathrm{X}(2 \mathrm{r}-3, \mathrm{c})+2 \mathrm{X}(2 \mathrm{r}-2, \mathrm{c})+6 \mathrm{X}(2 \mathrm{r}- \\
& 1, \mathrm{c})+2 \mathrm{X}(2 \mathrm{r}, \mathrm{c})-\mathrm{X}(2 \mathrm{r}+1, \mathrm{c}) \\
& \mathrm{m}\left(2^{\text {nd }} \text { Half }\right) \leftarrow-\mathrm{X}(2 \mathrm{r}-1, \mathrm{c})+2 \mathrm{X}(2 \mathrm{r}, \mathrm{c})-\mathrm{X}(2 \mathrm{r}+1, \mathrm{c}) \\
& \mathrm{n}\left(1^{\text {st }} \text { Half }\right) \leftarrow-\mathrm{Y}(2 \mathrm{r}-3, \mathrm{c})+2 \mathrm{Y}(2 \mathrm{r}-2, \mathrm{c})+6 \mathrm{Y}(2 \mathrm{r}-
\end{aligned}
$$


$\mathrm{n}\left(2^{\text {nd }}\right.$ Half $) \leftarrow-\mathrm{Y}(2 \mathrm{r}-1, \mathrm{c})+2 \mathrm{Y}(2 \mathrm{r}, \mathrm{c})-\mathrm{Y}(2 \mathrm{r}+1, \mathrm{c})$

ii) Inverse Row Transformation

For every row

The first column

$$
\mathrm{x}\left(1^{\text {st }} \text { Half }\right) \leftarrow 6 \mathrm{~W}(\mathrm{r}, 2 \mathrm{c}-1)+2 \mathrm{~W}(\mathrm{r}, 2 \mathrm{c})-\mathrm{W}(\mathrm{r}, 2 \mathrm{c}+1)
$$$$
\mathrm{x}\left(2^{\text {nd }} \text { Half }\right) \leftarrow-\mathrm{W}(\mathrm{r}, 2 \mathrm{c}-1)+2 \mathrm{~W}(\mathrm{r}, 2 \mathrm{c})-\mathrm{W}(\mathrm{r}, 2 \mathrm{c}+1)
$$

While last column

$$
\begin{aligned}
& \mathrm{x}\left(1^{\text {st }} \text { Half }\right) \leftarrow-\mathrm{W}(\mathrm{r}, 2 \mathrm{c}-3)+2 \mathrm{~W}(\mathrm{r}, 2 \mathrm{c}-2)+6 \mathrm{~W}(\mathrm{r}, 2 \mathrm{c}- \\
& \quad 1)+2 \mathrm{~W}(\mathrm{r}, 2 \mathrm{c}) \\
& \mathrm{x}\left(2^{\text {nd }} \text { Half }\right) \leftarrow-\mathrm{W}(\mathrm{r}, 2 \mathrm{c}-1)+2 \mathrm{~W}(\mathrm{r}, 2 \mathrm{c}) \\
& \text { and remaining columns } \\
& \mathrm{x}\left(1^{\text {st }} \text { Half }\right) \leftarrow-\mathrm{W}(\mathrm{r}, 2 \mathrm{c}-3)+2 \mathrm{~W}(\mathrm{r}, 2 \mathrm{c}-2)+6 \mathrm{~W}(\mathrm{r}, 2 \mathrm{c}- \\
& 1)+2 \mathrm{~W}(\mathrm{r}, 2 \mathrm{c})-\mathrm{W}(\mathrm{r}, 2 \mathrm{c}+1) \\
& \mathrm{x}\left(2^{\text {nd }} \text { Half }\right) \leftarrow-\mathrm{W}(\mathrm{r}, 2 \mathrm{c}-1)+2 \mathrm{~W}(\mathrm{r}, 2 \mathrm{c})-\mathrm{W}(\mathrm{r}, 2 \mathrm{c}+1)
\end{aligned}
$$

\subsection{Algorithm for Encryption}

Encryption is the means of transforming the image into indecipherable form. As security is concern for digital images, encrypting protects image content from malicious attacks. For encryption two different keys are considered: i) Symmetric (or) private key ii) asymmetric (or) public and private key. In the first case, single key is used for encryption and decryption while separate keys are used in the second case. The encryption algorithm includes the following steps.

Step1: Initialize the 8-bit cover image I and acquire its dimension $\mathrm{m} \times \mathrm{n}$.

Step 2: Generate the symmetrical key to be shared.

Step 3: Generate the random sequence using symmetrical key, denoted as $r$.

Step4: Perform bit wise operation for the input image I and random sequence $r$ given as:

$I_{1}=I \oplus r$, where $I_{1}$ is the Ciphered matrix

Step 5: Repeat step 2- 4 for all pixels.

It is adapted exact reverse process for decryption. Fig. 2 shows the Lena and its encrypted and decrypted images.

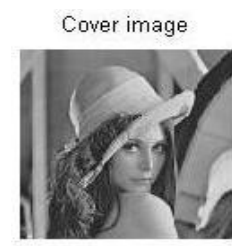

(a)

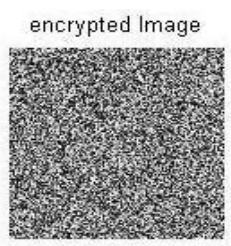

(b)

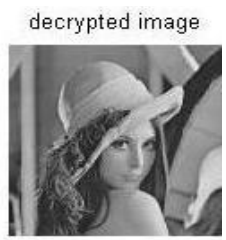

(c)
Fig 2: a) Lena b) encrypted form c) decrypted image

\subsection{Data Hiding Algorithm}

Once the encryption part was done, the hider starts embedding data as follows:

Step1: Read the image $I_{1}$ of size $\mathrm{h} \mathrm{x}$ w.

Step2: Segment $I_{1}$ into smaller blocks of size $\mathrm{p} \mathrm{x} \mathrm{p} \mathrm{such} \mathrm{that}$

$$
(u-1) \cdot \mathrm{p}+1 \leq h \leq u \cdot \mathrm{p}
$$

$$
(v-1) \cdot \mathrm{p}+1 \leq \mathrm{w} \leq v \cdot \mathrm{p}
$$

Where $u$ and $v$ are positive integers.

Step 3: Divide $p^{2}$ pixels of every block as $p_{o}$ and $p_{1}$ randomly using hiding key.

Step 4: If binary 0 is to be inserted, then change pixels in $p_{o}$ by three positions.

$$
I_{1}{ }^{\prime}=\overline{I_{h, w, k}},(h, w) \epsilon p_{o} \text { and } k=0,1,2
$$

Step 5: If binary 1 is to be inserted, then change pixels in $p_{1}$ by three positions.

$$
I_{1}{ }^{\prime}=\overline{I_{h, w, k}},(h, w) \epsilon p_{1} \text { and } k=0,1,2
$$

Other encrypted bits remain unchanged.

\subsection{Extraction algorithm}

After the embedding process is done, the extraction of embedded data is explained in the following steps:

Step 1: Apply discrete wavelet transform for the stego image and obtain four sub-bands as LL, HL, LH and $\mathrm{HH}$.

Step 2: perform zigzag scan order for HL, LH and HH.

Step 3: Exclusive-OR operation is performed between random sequence generator $r$ and decrypted image denoted as $I_{1}{ }^{\prime}$.

Step 4: If the inserted bit is 0 and belongs to pixel $p_{o}$, then the 3 LSB positions are decrypted as:

$$
I^{\prime}=r \oplus I_{1}{ }^{\prime}=r \oplus \overline{I_{h, w, k}}=r \oplus \overline{I \oplus r}=\bar{I}
$$

Step 5: If the inserted bit is 1 and belongs to pixel $p_{1}$, then the bits are decrypted as in step 4.

Step 6: Repeat step 3- 5 till embedded data is extracted.

\section{SIMULATION RESULTS}

The simulation analysis and the results for the proposed methodology is found to be effective for 512 X 512 cover images of "Lena", "Airplane", "Barbara", "Baboon", "Boat" and "Peppers" are shown in Fig. 3.

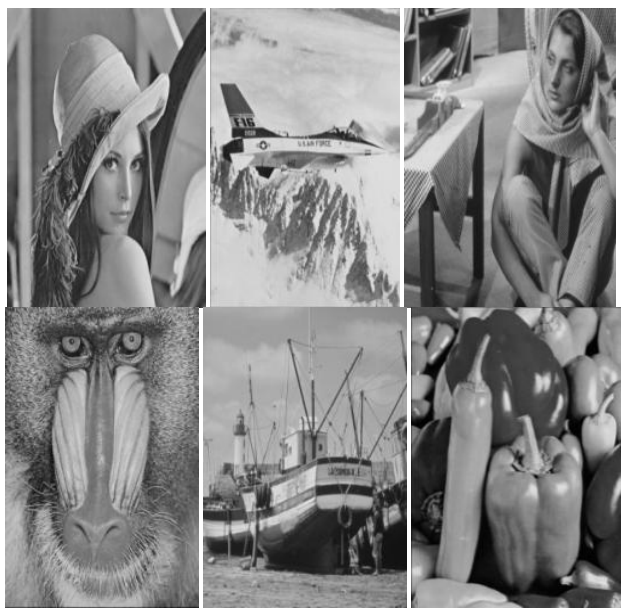

Fig 3: Cover Images used for simulation

In this study the parameter used to measure the visual quality with respect to the stego image is PSNR. The PSNR of any resultant image can be calculated using MSE is given in eq (7)

$P S N R=20 \log _{10}\left(\frac{255}{M S E}\right)$ 
Where

$M S E=\frac{1}{M x N} \sum_{i=1}^{M} \sum_{j=1}^{N}\left(I(i, j)-I^{\prime}(i, j)\right)^{2}$

$I(i, j)$ - cover image and $I^{\prime}(i, j)$ - Reconstructed image

The tradeoff in payload and PSNR is a drawback for existing methods. Which can be overcome by designing a 13/9 lifting scheme with 13 low pass and 9 high pass filters and hiding data up to 1, 31,072 bits with PSNR 58.17 dB for Lena. The PSNR versus bit rates for different cover images are presented in Table 1. The payload for different images at the bit rate of 0.5 bits per pixel are presented in Table 2 .

From table 1 and table 2 it is observed, for airplane image number of pixels embedded are high with high PSNR and for pepper image payload is high with PSNR comparatively high compared to other images except for airplane. From the table, it is also observed that higher the embedding capacity, higher is the PSNR value which is improved than the state-of-art methods. The 13/9 lifting scheme tested for six different images and the state-of-art methods as described for performance parameters of embedding rate versus PSNR is shown below in Fig 4 (a-f).

Table 1. PSNR values versus bit rates

Image PSNR Values for various Bit Rate (bpp)

\begin{tabular}{lcccccc}
\hline & 0.5 & 0.4 & 0.3 & 0.2 & 0.1 & 0.05 \\
Lena & 47.18 & 47.80 & 49.15 & 51.74 & 54.76 & 58.17
\end{tabular}

\begin{tabular}{lllllll} 
Airplane & 46.21 & 47.57 & 49.12 & 51.38 & 55.22 & 57.23 \\
\hline Barbara & 37.72 & 40.72 & 43.97 & 47.29 & 51.35 & 53.89
\end{tabular}

$\begin{array}{lllllll}\text { Baboon } & 38.13 & 40.57 & 43.05 & 45.85 & 50.85 & 54.60 \\ \text { Boat } & 44.78 & 46.42 & 48.62 & 50.80 & 53.25 & 55.50\end{array}$

Boat

$\begin{array}{lllllll}\text { Peppers } & 42.38 & 44.95 & 47.91 & 50.58 & 54.41 & 57.00\end{array}$

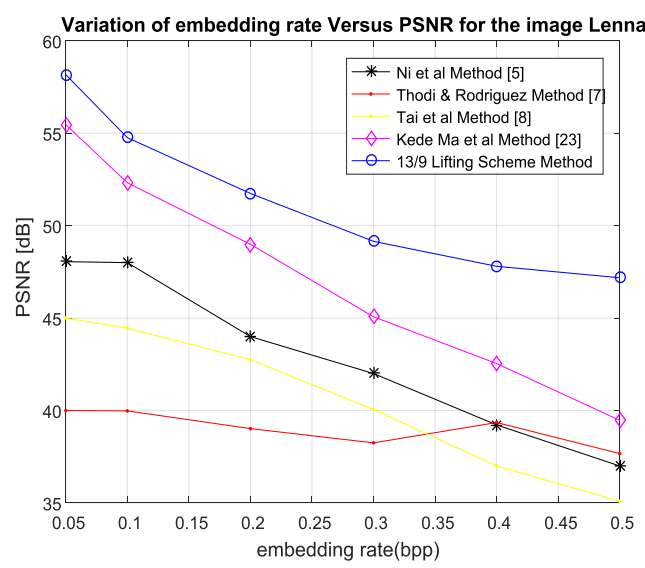

(a)
Table 2. Payload for different images at 0.5 bit rate

\begin{tabular}{|l|l|l|}
\hline Images & Bit Rate & Payload (bits) \\
\hline Lena & & 126034 \\
\cline { 1 - 1 } & & 131072 \\
Airplane & \multirow{4}{*}{$0.5 \mathrm{bpp}$} & 131072 \\
& & 131046 \\
\hline Baboon & & 131072 \\
\hline Boat & & 130206 \\
\hline
\end{tabular}

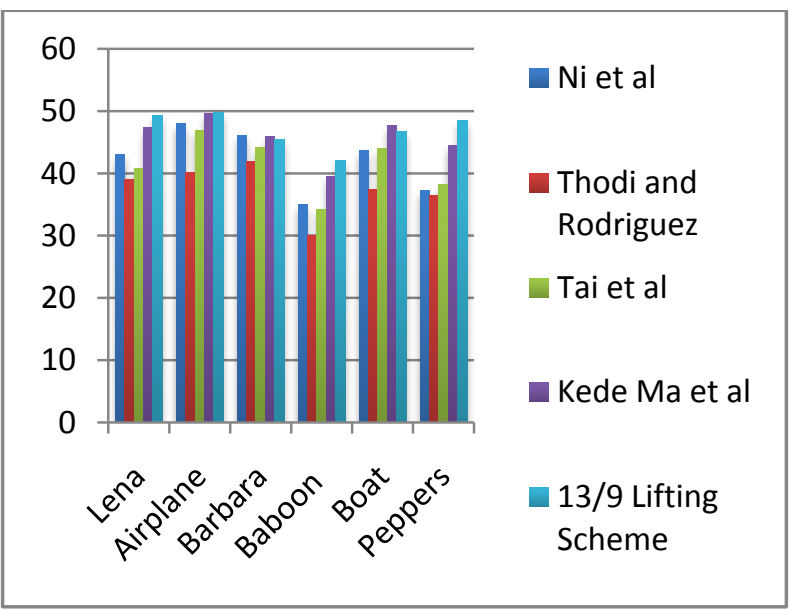

Fig 5: Average PSNR for proposed and existing methods

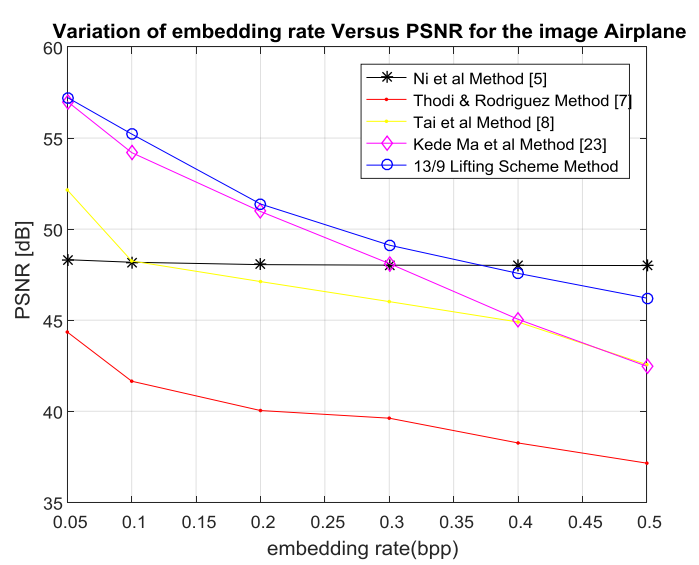

(b) 


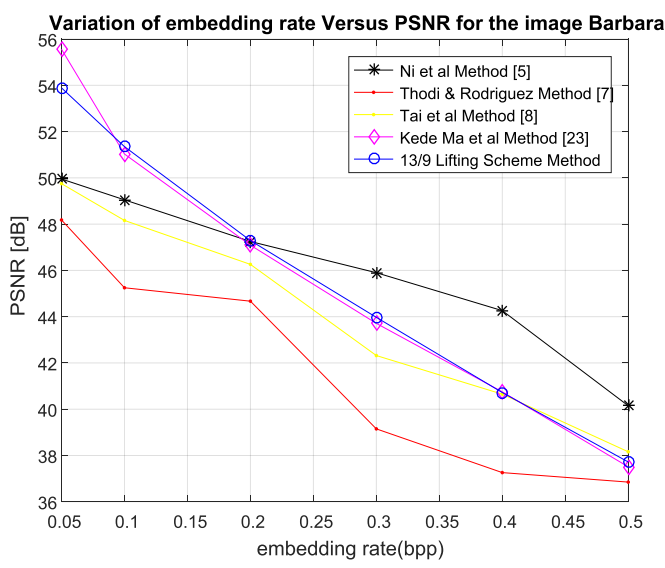

(c)

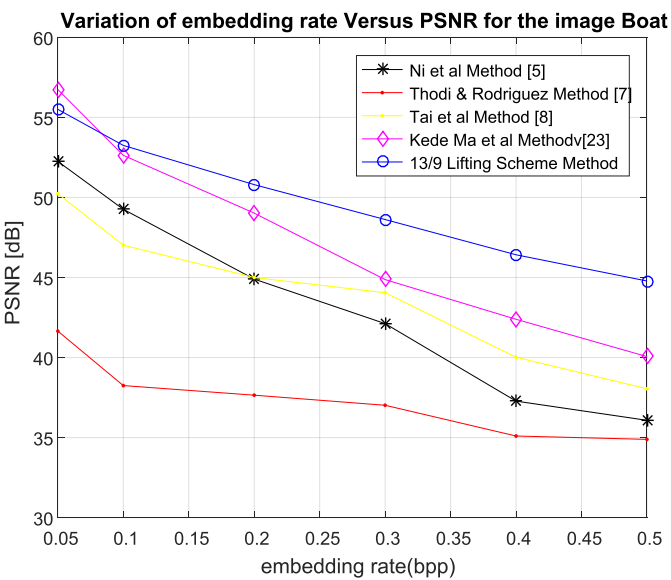

(e)

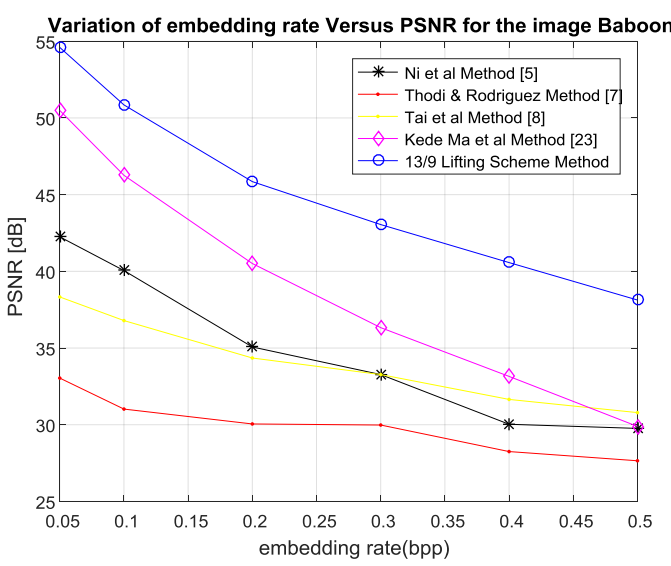

(d)

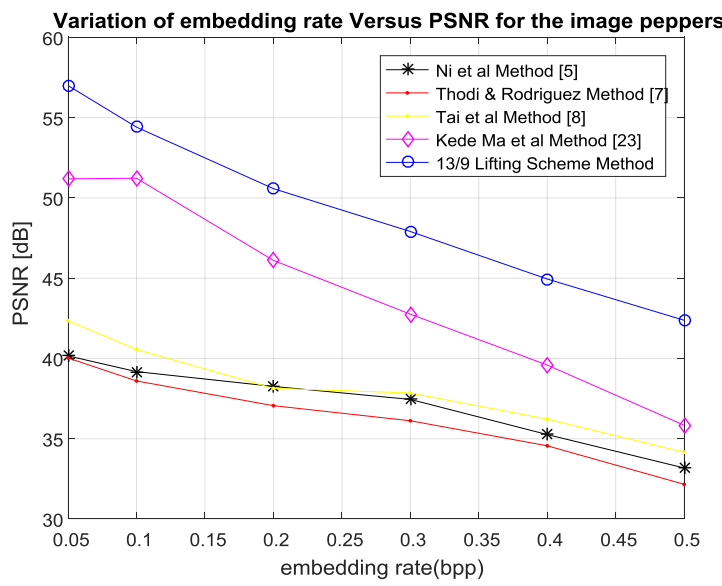

(f)

Fig 4: Comparison of Embedding Rate versus PSNR over six different images: a) "Lena" b) "Airplane" c) "Barbara" d) "Baboon" e) "Boat" f) "Peppers.

Table 3. Average PSNR of the proposed and existing methods for six cover images.

\begin{tabular}{|l|l|l|l|l|l|l|}
\hline \multirow{2}{*}{ Method } & \multicolumn{4}{|l|}{ Average PSNR (dB) } \\
\cline { 2 - 8 } & Lena & Airplane & Barbara & Baboon & Boat & Peppers \\
\hline Ni et.al & 43.04 & 48.09 & 46.09 & 35.07 & 43.64 & 37.24 \\
\hline Thodi et.al & 39.04 & 40.17 & 41.89 & 29.99 & 37.42 & 36.42 \\
\hline Tai et.al & 40.72 & 46.83 & 44.21 & 34.19 & 44.06 & 38.21 \\
\hline Kede Ma et.al & 47.31 & 49.64 & 45.94 & 39.44 & 47.62 & 44.45 \\
\hline 13/9 Lifting Scheme & 51.46 & 51.12 & 45.82 & 45.50 & 49.89 & 49.53 \\
\hline
\end{tabular}

From Fig 4 (a)-(f) the results are compared with other RDH methods of Ni et al. (2006), Thodi and Rodriguez (2007), Tai et al. (2009) and Kede Ma et al. (2013). These methods either use Difference Expansion (DE) or the histogram-based scheme for single embedding. Histogram based scheme gives high image quality while limiting the payload where as in DE scheme higher embedding capacity is possible with image distortion compared to histogram schemes. Fig 4 presents the results of embedding rate versus image quality and makes clear interpretation that proposed method achieved high visual quality with better embedding capacity. Taking the image "Lena" into consideration, it has attained higher visual quality of $58.17 \mathrm{~dB}$ for embedding rate of $0.05 \mathrm{bpp}$ and still maintained PSNR of $47.18 \mathrm{~dB}$ for 0.5 bpp. The 13/9 lifting scheme out performs well for all the six test image in embedding capacity and image quality compared to other state-of-art methods.

Table 3 presents the results of the average PSNR for six test images and Figure. 5 show the bar graph representation for the proposed method compared with the existing methods. It is observed that the average PSNR for Lena is $51.46 \mathrm{~dB}$ which is higher than the other existing methods. Except for the image Barbara, for all other images our method is superior in PSNR and hiding capacity than other existing methods. The 
reason is as it contains large areas of complex textures which are to be further rectified.

\section{CONCLUSION}

In this section results are summarized. Firstly, a novel 13/9 lifting scheme was implemented with 13 low pass and 9 high pass filters and then embedded data into that image using hiding algorithm and extracted the data from the images without distortion. When compared to state-of-art methods our method out performed well in payload as well as image quality with PSNR up to $58.17 \mathrm{~dB}$ and the average PSNR is larger than the existing methods which is equal to 45.50 $51.46 \mathrm{~dB}$ for all the test images. The simulation results shows that the proposed 13/9 lifting scheme gives better results in terms of the embedding capacity and PSNR value. In future this work can be extended with embedding rates greater than 1.5 bpp with better image quality.

\section{REFERENCES}

[1] J. Tian, "Reversible data embedding using a difference expansion", IEEE Trans. Circuits Syst.Video Technol., Vol. 12, No. 8, pp. 890-896, 2003

[2] Adnan M. Alattar, "Reversible Watermark Using the Difference Expansion of a Generalized Integer Transform", IEEE Trans On Image Processing., Vol. 13, No. 8, pp. 1147-1156, 2004.

[3] Yongjian Hu, Heung-Kyu Lee, and Jianwei Li, "DEBased Reversible Data Hiding With Improved Overflow Location Map", IEEE Transactions. Circuits and Systems for Video Technology., Vol. 19, No. 2, pp. 250260, 2009.

[4] Ju-Yuan Hsiao, Ke-Fan Chan, J.Morris Chang, "Blockbased reversible data embedding", Signal Processing., pp. 556-569, 2008

[5] Ni Z, Shi Y. Q, Ansari N. and Su W, "Reversible Data Hiding", IEEE Transactions. Circuits and Systems for Video Technology., Vol. 16, No. 3, pp. 354-362, 2006.

[6] H.-W. Tseng and C.-P. Hsieh, "Reversible data hiding based on image histogram modification", The Imaging Science Journal., Vol. 56, pp. 271-278, 2008.

[7] Thodi D.M, Rodríguez J.J, "Expansion embedding techniques for reversible watermarking", IEEE Transactions on Image Processing, Vol. 16, No. 3, pp. 721-730, 2007.

[8] W.L. Tai, C.M. Yeh, and C.C. Chang, "Reversible data hiding based on histogram modification of pixel differences", IEEE Trans. Circuits Syst. Video Technol., Vol. 19, pp. 906-910, 2009.

[9] Piyu Tsai , Yu-Chen Hu, Hsiu-LienYeh, "Reversible image hiding scheme using predictive coding and histogram shifting", Signal Processing., pp. 1129-1143, 2008

[10] Vasiliy Sachnev, Hyoung Joong Kim, Jeho Nam, Sundaram Suresh, and Yun Qing Shi, "Reversible Watermarking Algorithm Using Sorting and Prediction", IEEE Transactions. Circuits and Systems for Video Technology., Vol. 19, No. 7, pp. 989-999, 2009.
[11]Chin-Feng Lee, Hsing-Ling Chen, Hao-Kuan Tso, "Embedding capacity raising in reversible data hiding based on prediction of difference expansion", The Journal of Systems and Software.,pp. 1864-1872, 2010.

[12] Xiaolong Li , Jian Li , Bin Li , Bin Yang, "High-fidelity reversible data hiding scheme based on pixel-valueOrdering and prediction-error expansion", Signal Processing., pp. 198-205, 2012.

[13] Bo Ou, Xiaolong Li , Yao Zhao, Rongrong Ni, "Reversible data hiding based on PDE predictor",The Journal Of Systems and Software., pp. 2700-2709, 2013.

[14] Lixin Luo, Zhenyong Chen, Ming Chen, Xiao Zeng, and Zhang Xiong, "Reversible Image Watermarking Using Interpolation Technique",IEEE Transactions On Information Forensics and Security, Vol. 5, No. 1, pp. 187-193, 2010.

[15] Xing-Tian Wang, Chin-Chen Chang, Thai-Son Nguyen and Ming-Chu Li, "Reversible data hiding for high quality images exploiting interpolation and direction order mechanism", Digital Signal Processing., pp. 569577, 2012.

[16] Y. Yalman and F. Akar, "A high-capacity reversible data hiding method: HCRHide", The Imaging Science Journal., Vol. 62, No.2, pp. 121-126, 2013.

[17] Chia-Chen Lin and Pei-Feng Shiu, "High Capacity Data Hiding Scheme for DCT-based Images", Journal of Information Hiding and Multimedia Signal Processing., Vol.1, No.3, pp. 220-240, 2010.

[18] Fei Peng, Xiaolong Li, Bin Yang, "Adaptive reversible data hiding scheme based on integer transform", Signal Processing., pp. 54-62, 2011.

[19] Ching-Yu Yang, Chih-Hung Lin and Wu-Chih $\mathrm{Hu}$, "Reversible Data Hiding for High-Quality Images Based on Integer Wavelet Transform", Journal of Information Hiding and Multimedia Signal Processing., Vol.3, No.2, pp. 142-150, 2012.

[20] Yih-Kai Lin, "High capacity reversible data hiding scheme based upon discrete cosine transformation", The Journal of Systems and Software., pp. 2395-2404, 2012.

[21] Engin Avci, Turker Tuncer, Derya Avci, "A Novel Reversible Data Hiding Algorithm Based on Probabilistic XOR Secret Sharing in Wavelet Transform Domain", Arab J Sci Eng, Research Article - Computer Engineering and Computer Science., 2016.

[22] Fan Li, Qian Mao and Chin-Chen Chang, "Reversible data hiding scheme based on the Haar discrete wavelet transform and interleaving prediction method", Multimed Tools Appl., 2017.

[23] Kede Ma, Weiming Zhang, Xianfeng Zhao, Nenghai Yu and Fenghua Li, "Reversible data hiding in encrypted images by reserving room before encryption", IEEE Trans. Information forensics and security, , Vol. 8, pp. 553-562, 2013

[24] I. Daubechies and W. Sweldens, "Factoring Wavelet Transforms into Lifting Schemes", The Journal of Fourier Analysis and Applications, Vol. 4, pp. 247-269, 1998.

[25] Chengyi Xiong, Jinwen Tian and Liu, Jian, "Efficient Architectures for Two-Dimensional Discrete Wavelet Transform Using Lifting Scheme", IEEE Transactions on Image Processing, Vol.16, No.3, pp. 607-614, 2007. 